\section{Coagulation tests: what does this all mean?}

\section{Holly Witchell}

Primary haemostasis is where platelets form a plug sticking to the vessel walls to stem haemorrhage, secondary haemostasis is where clotting factors are released creating a cascade effect to result fibrin production, which reinforces the platelet plug to form a clot.

It is important to understand when to carryout coagulation tests on patients. There are many reasons for a patient to present with a coagulopathy, including sepsis, trauma, congenital disorders, ingested toxin, neoplasia or immune-mediated causes.

In-house tests help us get an idea of coagulation status quickly. Blood samples should be obtained from a peripheral vein so pressure can be easily applied to aid in haemostasis if a coagulopathy is present. Blood should be collected and placed into a citrate tube and mixed thoroughly but gently. Most tests should be run within 2 hours of sampling.

There are many different types of point-of-care coagulation tests available and they differ in cost, accuracy and result. Tests that aid in diagnosis of primary haemostasis disorders are looking at quantity and function of platelets: performing a blood smear and buccal mucosa bleeding time (BMBT). Tests used to check secondary haemostasis assess the function and amount of clotting factors; these tests are prothrombin time (PT), activated partial thromboplastin time (aPTT) and activated clotting time (ACT).

PT tells us about the extrinsic (factor VII) and common (factors I, II, V, X) coagulation pathways. PT is the first test to show an abnormality within the coagulation cascade due to factor VII having such a short half-life. A prolonged PT can mean there is a vitamin $K$ deficiency, as vitamin $\mathrm{K}$ aids in synthesis of factors II, VII, IX, X. Prolongation can also be due to a severe hepatopathy or disseminated intravascular coagulation.

The aPTT tells us about intrinsic (VIII, IX, XI, XII) and common coagulation pathways. A prolonged aPTT can be due to a congenital disease or coagulopathy, for example, factor VIII deficiency (haemophilia A), IX deficiency (haemophilia B) or von Willebrand's disease (vWD) due to von Williebrand's factor (vWF) deficiency.

$\mathrm{ACT}$ tests the same as the APTT but is less sensitive. Over $90 \%$ of the clotting factors need to be lost in order for ACT to be prolonged, whereas for PT and APTT are prolonged when over $70 \%$ of clotting factor is lost.

\section{KEY LEARNING OBJECTIVES}

- Be able to select the appropriate test required for the patient

Understand what each test is used for

- Understand basic coagulation pathways

\section{MULTIPLE CHOICE QUESTIONS}

1. A fall in the level of which clotting factor would be identified first in a rodenticide poisoning, due to having a short half-life?
(A) Factor VIII
(C) Factor VII
(B) Factor II
(D) Factor $V$

2. A 6-month-old Dobermann is being admitted for castration. Which test would be most appropriate to test for $\mathrm{WWD?}$
(A) PIVKA
(C) $\mathrm{PT}$
(B) $\mathrm{aPTT}$
(D) $\mathrm{ACT}$

3. Which test would you perform to check platelet function?
(A) Blood smear
(C) $\mathrm{aPTT}$
(B) $\mathrm{ACT}$
(D) BMBT

\section{Hypotension: cause, effect and management}

\section{Holly Witchell}

Blood pressure is an important parameter to monitor in our critical patients. It gives us information regarding tissue perfusion within the body and, if blood pressure is too low or too high, it leads us to investigate the cause and its knock-on effects on organ function and delivery of oxygen and nutrients to tissues.

Blood pressure is determined by cardiac output (CO). $\mathrm{CO}$ is dependent on heart rate and stoke volume. Stroke volume is made up of three components within the heart: preload, afterload and contractility. Without an effective 'pump' pushing the blood around the body the reduction in effective $\mathrm{CO}$ results in hypotension, which may cause ischaemia in organs and tissues.

Systemic vascular resistance (SVR) also has effects on blood pressure. This involves the vessel walls, whether they are constricted or dilated, and viscosity of the blood. These changes can be significant in critical patient, especially in cases like sepsis, where patients are vasodilated resulting in reduced SVR and hypotension.

Systolic blood pressure (SBP) is measured during ventricular contraction and diastolic blood pressure (DBP) is measured upon relaxation. Mean arterial blood pressure (MAP) is an average of pressures over a cardiac cycle and can be measured easily by oscillometric blood pressure monitor/machine or can be calculated if using a Doppler: $[\mathrm{MAP}=\mathrm{DBP}+(\mathrm{SBP}-\mathrm{DBP}) / 3]$. Doppler and oscillometric are non-invasive ways of measuring blood pressure. 
Indications of poor perfusion can also be monitored by examining pulse quality, mucous membrane colour, capillary refill time, heart rate, lactate, packed cell volume/total solids and acid-base status. These should be used in conjunction with blood pressure measurements.

Invasive blood pressure measurements are taken from a catheter placed directly into the artery, which is set up to a multiparameter monitor, giving a direct BP measurement and showing any changes in the patient's waveforms. Invasive monitoring has risks of catheter site infections and thrombosis, so the catheter should be handled aseptically.

\section{TREATMENT FOR HYPOTENSION}

Intravascular fluid boluses should be administered prior to drug therapies, $10-20 \mathrm{ml} / \mathrm{kg}$ bolus adds to the intravascular volume of the patient if the hypotension is due to hypovolaemia. The fluid bolus may have to be repeated but with caution: respiratory rate and effort should be monitored after each bolus to prevent potential pulmonary oedema due to fluid overload. If fluid boluses are not improving blood pressure, we need to start looking into drug therapies like vasopressors which increase SVR causing vasoconstriction and therefore increasing blood pressure and perfusion to vital organs and tissues.

\section{KEY LEARNING OBJECTIVES}

- Learn how hypotension is caused

- Understand how hypotension can be corrected
- Understand the effects hypotension can have on the body

\section{MULTIPLE CHOICE QUESTIONS}

1. What are the three components that make up stroke volume?

(A) Preload, afterload and contractility

(B) Systolic blood pressure, diastolic blood pressure and heart rate

(C) Heart rate, contractility and systolic blood pressure

(D) Cardiac output, preload and afterload

2. When is systolic blood pressure measured?

(A) During relaxation of the ventricles

(B) During contraction of the ventricles

(C) During one cardiac cycle giving us an average measurement

(D) During vasodilation

3. Which parameter should be monitored when administering fluid boluses to prevent fluid overload?
(A) Capillary refill time
(B) Heart rate
(C) Respiratory rate and effort
(D) Mucous membranes 